Die Ueberführung der Lävulose in ihr charakteristisches Methylphenylosazon gelingt so leicht, dass diese Methode mit. Vortheil zur Charakterisirung dieses Zuckers in den Producten des normalen und pathologischen Stoffwechsels dienen kann, wo sein Nachweis bislang mit grosser Unsicherheit behaftet war.

Ueber die Anwendung des Verfabrens auf physiologische Probleme und über einige kleine Modificationen, die durch die Besonderheit der in Betracht kommenden Materialien geboten sind, werde ich an anderer Stelle in Gemeinschaft mit H. Strauss berichten.

147. C. Harries und G. Hans Müller: Ueber die Condensation von Aethylmethylketon mit Benzaldehyd.

[Aus dem I. chem. Universitātslaboratorium zu Berlin.]

(Eingegangen am 12. Februar 1902.)

Während sich die Condensation von Aceton mit Benzaldehyd theoretisch nur in einem Sinne abspielen kann und auch in Wirklichkeit so verläuft ${ }^{1}$ ), lassen sich bei der Condensation von Aethylmethylketon mit Benzaldebyd mindestens zwei Producte voraussehen, welche durch Zusammentritt eines Moleküls Benzaldehyd mit einem Molekül Aethylmethylketon entsteben können.

$\alpha-\mathrm{C}_{6} \mathrm{H}_{5} . \mathrm{CH}: \mathrm{CH} . \mathrm{CO} . \mathrm{CH}_{2} . \mathrm{CH}_{3}, \gamma-\mathrm{C}_{6} \mathrm{H}_{5} . \mathrm{CH}: \mathrm{C}\left(\mathrm{CH}_{3}\right) . \mathrm{CO} \cdot \mathrm{CH}_{3}$.

Thatsächlich werden auch zwei von einander verschiedene Benzalproducte erhalten. Das eine entsteht fast quantitativ beim Schütteln von Benzaldehyd mit Aethylmethylketon und verdünnter Natronlauge, das andere beim Sättigen eines Gemisches der beiden Componenten mit Salzsäuregas. Eine ganz ähnliche Beobachtung haben vor Kurzem Guido Goldschmiedt und K. Krezmar ${ }^{2}$ ) beim Phenylaceton gemacht. Sie haben mit Sicherheit nacbgewiesen, dass dem alkalischen Benzalcondensationsproduct die Formel $\mathrm{C}_{6} \mathrm{H}_{5} . \mathrm{CH}_{2} . \mathrm{CO} . \mathrm{CH}: \mathrm{CH} . \mathrm{C}_{6} \mathrm{H}_{5}$, dem sauren dagegen die Formel $\mathrm{C}_{6} \mathrm{H}_{5} . \mathrm{C} . \mathrm{CO} . \mathrm{CH}_{3}$ zukommt.

\title{
CH. $\mathrm{C}_{6} \mathrm{H}_{5}$
}

Soeben ist eine Dissertation von E. Lerinstein ${ }^{3}$ ) erschienen, in welcher deren Verfasser den Beweis zu fübren versucht hat, dass das Benzaläthylmetbylketon, welches bei Gegenwart von Natronlange entsteht, die Constitution $\mathrm{C}_{6} \mathrm{H}_{5} . \mathrm{CH}: \mathrm{C}\left(\mathrm{CH}_{3}\right) \cdot \mathrm{CO} \cdot \mathrm{CH}_{3}$ besitzt; die Bildung des isomeren Ketons in saurer Lösung ist nicht beobachtet

1) Claisen und Ponder, Ann. d. Chem. 223, 139.

2) Monatshefte 22, 659 [1901].

3) E. Levinstein, Inaug. Diss. Berlin, 29. Januar 1902. 
worden. Darnach würde die Reaction aber gerade umgekehrt beim Methyläthylketon wie beim Phenylaceton verlaufen. Wenn auch nicht ausgeschlossen ist, dass der Zusammentritt von Benzaldehyd mit einem aliphatischen Keton in anderer $W$ eise als mit einem aromatischen erfolgen kann, so darf jedenfalls der von Levinstein geführte Beweis sowohl in speculativer wie experimenteller Beziehung als nicht stichbaltig angesehen werden.

Derselbe lautet folgendermaassen: Bei der Reduction der beiden möglichen Benzäläthylmethylketone müssen durch Aufnahme von 2 Wasserstoffatomen folgende Verbindungen entstehen:

I. $\mathrm{O}_{6} \mathrm{H}_{5} \cdot \mathrm{CH}: \mathrm{C}\left(\mathrm{CH}_{3}\right) \cdot \mathrm{CO} \cdot \mathrm{CH}_{3} \rightarrow \mathrm{C}_{6} \mathrm{H}_{5} \cdot \mathrm{CH}_{2} \cdot \mathrm{C}\left(\mathrm{CH}_{3}\right) \mathrm{H} \cdot \mathrm{CO} \cdot \mathrm{CH}_{3}$ oder $\mathrm{C}_{6} \mathrm{H}_{5} \cdot \mathrm{CH}: \mathrm{C}\left(\mathrm{CH}_{3}\right) \cdot \mathrm{CH}(\mathrm{OH}) \cdot \mathrm{CH}_{3}$,

II. $\mathrm{C}_{6} \mathrm{H}_{3} . \mathrm{CH}: \mathrm{CH} . \mathrm{CO} \cdot \mathrm{CH}_{2} \cdot \mathrm{CH}_{3} \rightarrow \mathrm{C}_{6} \mathrm{H}_{3} \cdot \mathrm{CH}_{2} \cdot \mathrm{CH}_{2} \cdot \mathrm{CO} \cdot \mathrm{CH}_{2} \cdot \mathrm{CH}_{3}$ oder $\mathrm{C}_{6} \mathrm{H}_{5} . \mathrm{CH}: \mathrm{CH}$. $\mathrm{CH}(\mathrm{OH}), \mathrm{CH}_{2} \cdot \mathrm{CH}_{3}$.

Die Reductionsproducte I müssen bei der Oxydation neben aromatischen Säuren Essigsäure, die in II aber Propionsänre ergeben. Da die bei der Reduction mit Zinkstaub und Essigsänre erhaltene Substanz (Schmp. $71^{0}$ ) beim Erhitzen mit zziemlich concentrirter Salpetersäure im Einschlussrohr 8 Stunden auf $200^{\circ}$ Essigsäure thatsächlich liefert, so glaubt Levinstein den Beweis für I erbracht zu haben.

Nun kann erstens auch aus einem Körper der Formel II bei einer solchen Oxydation Essigsäure entsteben. Viel wichtiger ist indessen zweitens die Frage, ob Levinstein überhaupt einfache Hydrirungsproducte der Formel I oder II in den Händen gehabt hat. Dieselbe ist entschieden zu verneinen. Wie im experimentellen Theil gezeigt werden wird, entsteben analog wie beim Benzalaceton, bei der Reduction der beiden Methyläthylbenzalketone mit Natriumamalgam in saurer Lösung in einer Ausbeute von ea. $60 \mathrm{pCt}$. normaler Weise zwei isomere Benzylmethyläthylketone, welche ölig sind nod Ketoneigenschaften besitzen. Le vinstein beschreibt ein Product vom Schmp. $71^{\circ}$, welches er bei der Reduction des Alkali-Benzaläthylmethylketons mit concentrirter Essigsäure und Zinkstaub erhalten hat. Wie er selbst sagt, war die Substanz nicht rein, trotzdem hat er dieselbe zur Constitutionsbestimmung verwerthet. Wir haben bisher bei Wiederholung der von Levinstein angegebenen Reductionsmethode den Körper vom Schmp. $71^{0}$ nicht, dagegen leicht einen Körper vom Schmp. $168-169^{\circ}$ erhalten können, der sich auch als Nebenproduct bei der anderen Reductionsmethode, welche wir benutzt haben, bildet; es ist dies ein Ketonkörper der jedenfalls dimolekular 
ist und die Formel $\mathrm{C}_{22} \mathrm{H}_{26} \mathrm{O}_{2}$ besitzt. Derselbe entspricht dem Diphenyloctandion ron $\mathrm{Harries}$ und Eschenbach${ }^{1}$ ):

$$
\begin{aligned}
& \mathrm{C}_{6} \mathrm{H}_{5} \cdot \mathrm{CH} \cdot \mathrm{CH}_{2} \cdot \mathrm{CO} \cdot \mathrm{CH}_{3} \\
& \mathrm{C}_{6} \mathrm{H}_{5} \cdot \mathrm{CH} . \mathrm{CH}_{2} \cdot \mathrm{CO} \cdot \mathrm{CH}_{3}
\end{aligned}
$$

$Z_{n}$ bemerken ist, dass die Reduction mit Zinkstaub und Essigsäure bei den $\alpha, \not{\beta}$-ungesättigten Ketonen in der Regel analog wie diejenige mit Natriumamalgam in saurer Lösung verläuft ${ }^{2}$ ). Es ist bis jetzt noch nicht in einwandsfreier Weise gelungen, die Constitution der beiden Benzalmethyläthylketone aufzuklären, jedoch wird dies in kurzer Zeit sicher gelingen (vergl. Schlussbemerkung). Die Publication dieser noch nicht vollendeten Arbeit wurde durch das Erscheinen der erwähnten Dissertation veranlasst. Die mit alkalischen Mitteln entstehende Verbindung wird als $\alpha$, , die in saurer als $\gamma$-Benzalätbylmethylketon bezeichnet werden.

I. Darstellung des a-Benzal-äthylmethylketons.

$100 \mathrm{~g}$ Methyläthylketon und $140 \mathrm{~g}$ Benzaldehyd werden in $1500 \mathrm{~g}$ Wasser, dem $50 \mathrm{~g} \mathrm{10}$ procentige Natronlauge zugesetzt sind, suspendirt und 8 Tage auf der Maschine geschüttelt. Das Reactionsproduct. wird in Aether aufgenommen und nach dem Abdampfen des Letzteren im Vacuum fractionirt. Die Ausbeute an fractionirtem Rohproduct beträgt $200 \mathrm{~g}$.

Sdp. $142^{\circ}$ bei $12 \mathrm{~mm}$ Druck. Aus Petroläther glänzende Schuppen, Schmp. $38-39^{\circ}$ (Levinstein $39-40^{\circ}$ ), leicht löslich in Alkohol, Aether, Benzol, schwer löslich in Wasser.

Ein Nachlauf siedet bei $215-225^{\circ}$ und erstarrt za einem weissen Krystallbrei, der noch nicht näher untersucht wurde.

$0.2013 \mathrm{~g}$ Sbst.: $0.6088 \mathrm{~g} \mathrm{CO}_{2}, 0.1338 \mathrm{~g} \mathrm{H}_{2} \mathrm{O} .-0.1846 \mathrm{~g}$ Sbst.: $0.5571 \mathrm{~g}$ $\mathrm{CO}_{2}, 0.1217 \mathrm{~g} \mathrm{H}_{2} \mathrm{O}$.

$$
\begin{aligned}
& \mathrm{C}_{11} \mathrm{H}_{12} \mathrm{O} \text {. Ber. C 82.58, H 7.5, } \\
& \text { Gef. " } 82.48,82.31, \text { " 7.45, 7.40. }
\end{aligned}
$$

Das Oxim (Nadeln) sehmilzt bei $85-86^{\circ}$, das P henylhydrazon bei $101^{\circ}$. Schüttelt man das $a$-Benzaläthylmethylketon mit der äquivalenten Menge Benzaldehyd weiter, so entsteht ein weisser, krystallinischer Körper, dem nach Analyse und Eigenschaften die Formel eines Pyrons zukommt. Schmp. 68.5 . Auch dieses ist bereits ron Levinstein, aber anf anderem Wege erhalten worden.

$0.190 \mathrm{~g}$ Sbst.: $0.5661 \mathrm{~g} \mathrm{CO}_{2}, 0.1173 \mathrm{~g} \mathrm{H}_{2} \mathrm{O}$.

$$
\begin{aligned}
& \mathrm{C}_{18} \mathrm{H}_{18} \mathrm{O}_{2} \text {. Ber. C 81.20, H } 6.77 \text {. } \\
& \text { Gef. 》 } 81.26, \gg 6.92 \text {. }
\end{aligned}
$$

1) Diese Berichte 29, 380 [1896].

2) Vergl. Schneidewind, diese Berichte 21, 1325 [1888]; Harries u. Hübner, Ann. d. Chem. 296, 295 [1897]. 
Das Oxim schmilzt bei $195-196^{\circ}$.

Das $\alpha$-Monobenzaläthylmethylketon addirt in Eissessiglösung in der Kälte leicht 2 Atome Brom und liefert die Verbindung $\mathrm{C}_{6} \mathrm{H}_{5}$. CHBr . $\mathrm{CHBr}\left(\mathrm{C}_{3} \mathrm{H}_{5} \mathrm{O}\right)$, welche in kleinen Nadeln krystallisirt und bei $109-$ $110^{\circ}$ schwilzt.

Reduction des a-Benzal-äthylmethylketons.

Diese Verbindung lässt sich mit Natriumamalgam in saurer Lösung sehr leicht ohne jedwede Verharzung reduciren, wenn man die Methode anwendet, welche von C. Harries und seinen Mitarbeitern in einer grossen Anzahl von Untersuehungen ausgearbeitet worden ist. Es entstehen analog wie beim Benzalaceton $\left.{ }^{1}\right)$ zwei Producte:

I. $\mathrm{C}_{6} \mathrm{H}_{5} \cdot \mathrm{CH}: \mathrm{C}\left(\mathrm{C}_{3} \mathrm{H}_{6} \mathrm{O}\right) \rightarrow \mathrm{C}_{6} \mathrm{H}_{5} \cdot \mathrm{CH}_{2} \cdot \mathrm{CH}\left(\mathrm{C}_{3} \mathrm{H}_{6} \mathrm{O}\right)$, ein Benzylketon und

$$
\text { II. } 2 \mathrm{C}_{6} \mathrm{H}_{5} \cdot \mathrm{CH}: \mathrm{C}\left(\mathrm{C}_{3} \mathrm{H}_{6} \mathrm{O}\right) \longrightarrow \begin{aligned}
& \mathrm{C}_{6} \mathrm{H}_{3} \cdot \mathrm{CH} \cdot \mathrm{CH}\left(\mathrm{C}_{3} \mathrm{H}_{6} \mathrm{O}\right) \\
& \mathrm{C}_{6} \mathrm{H}_{5} \cdot \mathrm{CH} \cdot \mathrm{CH}\left(\mathrm{C}_{3} \mathrm{H}_{6} \mathrm{O}\right)
\end{aligned}
$$

Ersteres bildet sich in einer Ausbeute von ca. $60 \mathrm{pCt}$. Letzteres ron ca. $6 \mathrm{pCt}$.

Das $a-B e n z y l-m e t h y l a ̈ t h y l k e t o n(I), \mathrm{C}_{6} \mathrm{H}_{5} \cdot \mathrm{CH}_{2} \cdot \mathrm{CH}\left(\mathrm{C}_{3} \mathrm{H}_{6} \mathrm{O}\right)$, bildet ein farbloses, stark lichtbrechendes Oel, welches unter $17 \mathrm{~mm}$ Druck bei $128^{\circ}$, unter gewöhnlichem Druck bei $250-251^{\circ}$ siedet.

$$
\begin{gathered}
\text { Pefraetiometrische Messung: } \\
\mathrm{n}_{\mathrm{D}}^{20^{\theta}}=1.50882, \delta^{20^{\circ}}=0.9767 .
\end{gathered}
$$

Molekularrefraction. Ber. für Keton . . . . . . 49.633,

$$
\text { 》 "ungesättigten Alkohol . 50.5 T4, }
$$

Molekularrefraction. Gef. . . . . . . . . . . . 49.52 .

$0.1994 \mathrm{~g}$ Sbst.: $0.5931 \mathrm{~g} \mathrm{CO}_{2}, 0.1533 \mathrm{~g} \mathrm{H}_{2} \mathrm{O}$.

$$
\begin{aligned}
& \mathrm{C}_{11} \mathrm{H}_{14} \mathrm{O} \text {. Ber. C } 81.48, \mathrm{H} 8.64 \text {. } \\
& \text { Gef. 》 81.12, 》 } 8.62 \text {. }
\end{aligned}
$$

Das Keton giebt ein öliges $\mathrm{Oxim}$ und Phenylhydrazon. Letzteres geht in der Wärme mit Essigsäure in ein festes Product vom Schmp. $136^{\circ}$ aber, welches nach der Analyse durch Ammoniakverlust aus dem Phenylhydrazon entstanden ist. Vielleicht liegt ein Indolderivat vor.

\section{Bimolekulares Reductionsproduct (II).}

Krystallisirt aus dem Rohproduct der Reduction direct, aus Alkohol scheidet es sich in weissen Nadeln rom Schmp. $168-169^{\prime}$ ab.

$0.1607 \mathrm{~g}$ Sbst.: $0.4814 \mathrm{~g} \mathrm{CO}_{2}, 0.1161 \mathrm{~g} \mathrm{H}_{2} \mathrm{O}$.

$$
\begin{array}{r}
\mathrm{C}_{22} \mathrm{H}_{26} \mathrm{O}_{2} \text {. Ber. C 81.99, H } 8.07 \text {. } \\
\text { Gef. " } 81.7 \text {, " } 8.09 \text {. }
\end{array}
$$

Als Keton charakterisirt es sich durch Bildung eines Diphenylhydrazons.

1) loc. cit. 
II. Darstellung des $\gamma$-Benzal-äthylmethylketons.

$100 \mathrm{~g}$ Methyläthylketon wurden mit $140 \mathrm{~g}$ Benzaldehyd vermiseht and in der Kältemisehung trockne Salzsäure bis zur Sättigung eingeleitet. Dabei färbt sich das Reactionsgemisch roth, und nach 1-ztägigem Stehen ist die Einwirkung beendet. Das ölige Product wird mit verdünnter Natronlauge durchgeschültelt, mit Wasser gewaschen und in Aether aufgenommen. Der Aetherrückstand krystallisirt in grossen Nadeln, die eigenthünlich campherartig riechen. Aus Petrolăther ( $1 \mathrm{~g}$ in $8-9 \mathrm{ccm}$ bei 20 löslich) kommt das Keton in feinen Nadeln heraus, die ebenso wie das a-Keton bei $38^{\circ}$ schmelzen und wnter $12 \mathrm{~mm}$ Druek bei $127-130^{\circ}$ sieden. Gleiche Theile $\alpha-$ und p-Keton zusammengerieben, sehmelzen sofort.

Es ist möglich, dass sich als $Z_{\text {wischenglied ein Salzsäureadditions- }}$ product $\mathrm{C}_{11} \mathrm{H}_{13} \mathrm{OCl}$ bei dieser Condensation bildet, welches aber sehr leicht die Salzsäure verliert. Ausbeute quantitativ.

$0.1853 \mathrm{~g}$ Sbst.: $0.5574 \mathrm{~g} \mathrm{CO}_{2}, 0.1243 \mathrm{~g} \mathrm{H}_{2} \mathrm{O}$.

$\mathrm{C}_{11} \mathrm{~B}_{12} \mathrm{O}$. Ber. C 82.50, H 7.50.

Gef. $\$ 82.04, * 7.51$.

Das Phenylhydrazon, $\mathrm{C}_{11} \mathrm{H}_{12}: \mathrm{N} . \mathrm{NH} . \mathrm{C}_{6} \mathrm{H}_{5}$, selinilat, aus Alkohol umkrystallisirt, bei $105^{0}$ (unseharf). Das Oxim krystallisirt in grossen Prismen rom Sehmp. 103-1040.

Das Keton addirt in Eisessig unter den früher angegebenen Bedingungen zwei Atome Brom und bildet ein in Wasser unlösliches Di. bromderivat, welches sich sebr leicht zersetzt. Sehmp. $61^{\circ}$.

Die Reduction des $\gamma$-Benzal-ätbylmethyliketons führt unter den zuerst geschilderten Bedingungen in einer Ausbeute von ca. $60 \mathrm{pCt}$. zum $\gamma$-Benzyl-äthylmethylketon, welches unter $13 \mathrm{~mm}$ Drack bei $110-115^{\circ}$, unter gewöhnlichem Druck bei $234^{0}$ siedet.

$0.28405 \mathrm{~g}$ Sbst.: $0.7017 \mathrm{~g} \mathrm{CO}_{2}, 0.1849 \mathrm{~g} \mathrm{E}_{3} \mathrm{O}$.

$\mathrm{C}_{\mathrm{t}} \mathrm{H}_{44} \mathrm{O}$. Ber. C $81.48, \mathrm{H} 8.64$.

Gef. $\gg 81.70, \gg 8.85$.

Refractometrische Bestimmung:

$$
\delta 20^{\circ}=0.9751, \mathrm{n} \frac{20^{9}}{\mathrm{~T}}=1.50638 .
$$

Molekularrefraction. Ber. für Keton . ... . 49.63.

$$
\begin{gathered}
\gg \text { ungesăttigten Alkohol } 50.57 \text {. } \\
\text { Gef. . . . . . . . } \mathbf{4 9 . 4 4 .}
\end{gathered}
$$

In Gegensatz zum a-Benzal-äthylmetbylketon ist bei der Reduction der $/-$ Verbindung das dimolekulare Condensationsproduct $\mathrm{C}_{22} \mathrm{H}_{26} \mathrm{O}_{2}$ nur als Oel isolirt worden, welches ganz allmählich erstart. Sdp. $190-210^{\circ}$ unter $8 \mathrm{~mm}$ Druck.

Nach dem vollständigen Abschluss der Untersuchung wird eine ausführlichere Mittheilung über die Eigenschaften und Umwandlungen. ler beiden Ketone erfolgen. 
Nachschrift. Während der Drucklegung dieser Arbeit ist es uns gelungen, einen eindentigen Beweis für die Constitution der beiden Ketone zu erbringen. Wir erbielten nämlich durch trockne Destillation der Calciumsalze von Hydrozimmtsäure und Propionsäure in reichlicher Menge ein Keton, welches nach seiner Synthese als $\alpha$-Benzylmethyläthylketon anzusprechen ist

$\left(\mathrm{C}_{6} \mathrm{H}_{5} \cdot \mathrm{CH}_{2} \cdot \mathrm{CH}_{2} \cdot \mathrm{COO}\right) \mathrm{Ca}$ $\left(\mathrm{CH}_{3} \cdot \mathrm{CH}_{2}, \mathrm{COO}\right) \mathrm{Ca}$<smiles>CCOC(=O)CCc1ccccc1</smiles>

Dieses hat sich als rollkommen identiseh mit dem $\alpha$-Benzylmethyläthylketon erwiesen, welches wir durch Reduction des $\alpha$-Renzalmethyläthylketons gewonnen haben.

Daher kann man jetzt endgültig dem Condensationsproduct von Benzaldehyd mit Aethylmethylketon in alkaliseher Lösung die Formel $\left(\varepsilon-\mathrm{C}_{6} \mathrm{H}_{5} . \mathrm{CH}\right.$ : $\mathrm{CH} . \mathrm{CO} . \mathrm{CH}_{2} . \mathrm{CH}_{3}$, demjenigen mit Salzsäure die Formel $\left(\gamma-\mathrm{C}_{6} \mathrm{H}_{5} \cdot \mathrm{CH}: \mathrm{C}\left(\mathrm{CH}_{3}\right) \cdot \mathrm{CO} \cdot \mathrm{CH}_{3}\right.$ zuweisen. Die Verbältnisse liegen also gerade umgekehrt, wie Levinstein behauptet hat.

\section{M. Busch: Ueber die Constitution des Phenylurazols.}

(Eingegangen am 26. Februar 1902.)

Das jüngst erschienene Heft 2 dieser Berichte bringt unter dem gleichen Titel eine Abhandlung von S. F. Acree (S. 553), in der u. a. wieder die Constitution der Urazine erörtert wird; Hrn. Acree ist dabei entgangen, dass ich erst vor Jahresfrist ') nachgewiesen habe, dass die sogen. Urazine in Wirklichkeit Aminourazole sind.

Durch Methyliren des Phenylurazols erhält Acree eine Monomethylverbindung, identisch mit dem Methylphenylurazol, welches Heinrichs und ich ${ }^{2}$ ) aus dem Methyl-1-phenyl-4-aminourazol durch Eliminirung der Aminogruppe gewonnen baben, und in welchem wir s. Z. eine Methoxyverbindung (Formel I) vermutheten. Aus der Beobachtung, dass die betr. Substanz beim Eindampfen mit Salzsäure unverändert bleibt, zieht Acree den Schluss, dass eine $\mathrm{N}$-Methylver-

7) Chem. Centralblatt 1901, I, 933; diese Berichte 34, 2311 [1901]. Auch A. Purgotti und G. Vigano scheinen diese Untersuchungen nicht zu kennen, da sie neuerdings (Chem. Centralblatt 1902, I, 480) wieder die Urazin-Formel gebranchen.

2) Diese Berichte 33, 455 [1900]. 